\title{
Revisiting the approach to patients with stable ischaemic symptoms
}

\section{Anthony J. Dalby}

Life Fourways Hospital, Fourways, Randburg, South Africa

Address for correspondence:

Dr Anthony Dalby

Cardiologist

Life Fourways Hospital

Cnr Cedar Road and Cedar Avenue West

Fourways

Randburg

2191

South Africa

Email:

ajd@drdalby.co.za

The 2019 European Society of Cardiology Guideline on chronic coronary syndromes ${ }^{(1)}$ defines coronary artery disease (CAD) as the result of atherosclerotic plaque accumulation in the epicardial coronary arteries. The disease is chronic and progressive, characterised by long quiescent periods punctuated by acute exacerbations. The chronic coronary syndromes are categorised as (i) stable angina or dyspnoea, (ii) new-onset heart failure or left ventricular dysfunction and suspected CAD, (iii) stable symptomatic or asymptomatic patients less than I year after acute coronary syndrome or revascularisation, (iv) stable symptomatic or asymptomatic patients more than I year after diagnosis or revascularisation, (v) patients with vasospastic or microvascular angina, and (vi) asymptomatic patients whose CAD is detected at screening.

Since the introduction of coronary angioplasty by Dr Andreas

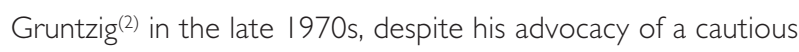
and conservative approach to coronary intervention, cardiologists seized on angioplasty and then later stents to treat patients with all manner of obstructed coronary arteries. A familiar clinical scenario would be the middle-aged smoker with dyslipidaemia who complains of angina pectoris twice a month and is shown to have ST segment depression in the ECG. Commonly, this patient will be offered coronary angiography and if a stenosis is found, the cardiologist, influenced by the "oculostenotic reflex" will proceed immediately to revas- cularisation by angioplasty and stenting. In most cases, the vessel can be successfully revascularised and the cardiologist confidently congratulates the patient with the assurance that a serious future cardiovascular event has been avoided.

Despite the visually satisfying effect of this approach, it is appropriate to question whether the intervention improved the patient's quality of life and chances of survival. This overview examines the results of the clinical trials in patients with stable ischaemic symptoms that have compared revascularisation with medical treatment.

\section{COMPARISON OF CORONARY BYPASS \\ SURGERY TO MEDICAL TREATMENT}

The Coronary Artery Surgery Study ${ }^{(3)}$ which was performed between 1975 and 1979 included 780 patients with stable coronary artery disease. These patients were assigned to either coronary bypass surgery or medical management. Excellent survival was reported in both groups whether single vessel, double vessel or triple vessel coronary disease was present. The authors concluded that patients like those enrolled in the trial could safely defer bypass surgery until symptoms worsened to the point that surgical palliation was required. Subgroup analysis ${ }^{(4)}$ showed that survival was improved by surgical revascularisation in patients with left main stem stenosis of $60 \%$ or greater, in particular those with moderate to severe impairment of left ventricular function. The Veterans Administration cooperative study of surgery for coronary arterial occlusive disease $^{(5)}$ drew similar conclusions. In their second interim report the European Coronary Surgery Study Group ${ }^{(6)}$ found that surgical revascularisation conferred significantly better 5 -year survival than those who were not operated. The subgroups of patients with left main disease and 3 vessel disease derived the greatest benefit. Consequently, even before the emergence of frequently performed percutaneous revascularisation, there was evidence that in many cases the relief of coronary obstruction did not confer an overall survival benefit.

\section{COMPARISON OF PERCUTANEOUS INTERVENTION OR CORONARY BYPASS SURGERY TO MEDICAL TREATMENT}

The COURAGE trial(7) was published in 2007. The result was widely criticised for the intense selection criteria applied which 
resulted in only $6.4 \%$ of the 35589 patients assessed for the trial being included. These were patients with stable angina who were randomised to either percutaneous intervention or medical therapy. Approximately 10\% of patients were lost to follow-up during the trial. Accepting these reservations, over 7 years of follow-up no difference could be shown when $\mathrm{PCl}$ was compared to medical therapy whether death from any cause and myocardial infarction, overall survival, survival free of acute coronary syndrome or survival free of myocardial infarction was considered.

Two years after the COURAGE trial, the BARI 2D Study Group $^{(8)}$ reported on 2368 patients with type II diabetes treated on insulin or oral drugs who had coronary artery disease proven on angiography. Patients were included if they had a positive exercise stress test with a coronary stenosis greater than $50 \%$ or alternately had classical angina with a coronary stenosis greater than $70 \%$. Based upon the extent of their coronary disease, approximately one third of patients were considered suitable for coronary bypass surgery while the remainder were deemed suitable for percutaneous coronary intervention. Half the patients suitable for coronary bypass surgery and half the patients suitable for percutaneous intervention were then randomised to receive initial medical therapy without revascularisation. These patients were followed for 5 years. During this period $42 \%$ of patients initially receiving only medical treatment underwent revascularisation. At 5 years there was no difference in the survival or the incidence of major cardiovascular events between the patients initially treated medically and those who had revascularisation in addition to medical treatment, whether they had had percutaneous coronary intervention or coronary bypass surgery. A higher mortality was noted in the bypass surgery group amongst which there was a higher incidence of triple vessel disease, proximal left anterior descending stenoses and chronic total occlusions. The conclusion drawn from this study was that a strategy of prompt coronary revascularisation in patients treated with intensive medical therapy for diabetes and stable ischaemic heart disease did not significantly reduce the rate of death from any cause or of major cardiovascular events. However, a later analysis of the trial $\left.\right|^{(9)}$ that quantified the severity of coronary disease according to the baseline SYNTAX score ${ }^{(10)}$ found that while percutaneous coronary intervention had no significant impact on long-term outcomes in those with a higher score, coronary bypass surgery plus medical therapy had resulted in an almost 50\% reduction in the incidence of death, myocardial infarction and stroke when compared to initial medical treatment only. The authors concluded that among patients with diabetes and stable ischaemic heart disease, higher
SYNTAX scores predicted higher rates of major cardiovascular events and, when compared to medical therapy, were associated with more favourable outcomes in those patients suitable for revascularisation by coronary bypass surgery.

In 2014, a large network meta-analysis of more than 90 trials that had compared revascularisation to medical treatment alone was published. (11) The authors reported significant reductions in mortality (20\%), myocardial infarction (21\%) and the need for revascularisation (84\%) with coronary bypass surgery when compared to medical treatment. The trials of percutaneous transluminal revascularisation that were included ranged from early studies with balloon angioplasty through bare metal stents to the newer generation drug-eluting stents. Though there were progressive reductions in the need for revascularisation with the development of improved stent technology (56\% - 73\%), overall there was no mortality benefit from percutaneous intervention nor was there a significant reduction in myocardial infarction. Considering only the newer generation drug-eluting stents (everolimus and zatarolimus), there was a reduction in mortality (25\%) compared to medical treatment. This analysis did not evaluate the extent of coronary artery disease present in these patients nor was the average period of follow-up stated.

\section{ISCHEMIA AND ISCHEMIA-CKD}

The International Study of Comparative Health Effectiveness with Medical And Invasive Approaches (ISCHEMIA) trial ${ }^{(12)}$ compared routine invasive therapy plus optimal medical therapy to optimal medical therapy alone in stable patients with moderate-to-severe ischaemia demonstrated on non-invasive stress testing with a nuclear study, echocardiographic stress testing, cardiac MRI or treadmill exercise testing. 5179 patients were randomised. The frequency of the patients' angina was daily or weekly in $22 \%$, several times a month in $44 \%$ and $34 \%$ did not experience angina. The majority of patients underwent CT coronary angiography to exclude the presence of left main stem stenosis. Patients with recent myocardial infarction, an ejection fraction below 35\%, NYHA class III or IV heart failure, unacceptable levels of baseline angina and those who had undergone revascularisation within the last year were also excluded. Patients with chronic kidney injury were assigned to a separate trial. Follow-up was for 3.3 years. In the invasive arm $96 \%$ of patients underwent cardiac catheterisation and $80 \%$ were revascularised. Over the trial period $28 \%$ on optimal medical therapy only were judged to be therapeutic failures and underwent coronary angiography. 23\% of the group were revascularised. The primary endpoint of the trial was a composite of cardiovascular death, myocardial infarction, resus- 
citated cardiac arrest, and hospitalisation for unstable angina or heart failure. The frequency of the primary endpoint was $13.3 \%$ in the invasive group and $15.5 \%$ in those on optimal medical therapy alone. The difference was not statistically significant. There was a threefold increase in peri-procedural myocardial infarction and a one third reduction in spontaneous myocardial infarction in the invasive arm. The Kaplan-Meier curves demonstrated a $2 \%$ increase in harm in the first 6 months in the invasive group and a $2 \%$ greater benefit at 4 years. The quality of life was improved in the invasive group only in those patients with angina.

The parallel ISCHEMIA-CKD trial(13) enrolled 777 patients with chronic kidney injury defined as an eGFR less than 30. The design was essentially like that of the main ISCHEMIA trial. The 3-year mortality in these patients was 25\%. Once again, there was no difference in outcome when invasive therapy plus optimal medical therapy was compared to optimal medical therapy alone. There were no substantial or sustained benefits regarding angina-related health status with an initially invasive strategy as compared with a conservative strategy.(14) The trialists concluded that intervention in patients with chronic kidney disease should be reserved for those who require relief of symptoms.

The following are the opinions of several experts regarding the impact of ISCHEMIA. These results were achieved using the very best medical therapy with the latest treatment targets, the best cobalt-chromium limus-eluting thin-strut stents, derivatives that reduce re-stenosis, and a modern-day approach to coronary bypass surgery (Antman E). ISCHEMIA proved that revascularisation does not have a marked effect on outcome (de Lemos J) and indicates that not every patient with moderate-to-severe ischaemia needs to go to the cath lab right away nor that not every blockage needs to have a stent right away (Nallamothu BJ). Presently most patients with chronic coronary syndrome land up in the cath lab where the cardiologist is expected to fix them (Mehran R). Stress tests will still be necessary to confirm that symptoms are arising from ischaemia (Poppas A) but rather than performing several stress tests, it is more important to rule out left main stem stenosis with $\mathrm{CT}$ coronary angiography (Mehran R). This change in emphasis in non-invasive testing is predicted to have major effects on future practice (de Lemos J). One would be comfortable advising patients not to undergo the invasive strategy if their angina were absent or controlled or tolerated. There should be no obligation to send them to the cath lab (Jacobs A). Naturally, interventionalists weighed in with critical comment, pointing out that the early difference in the trial was driven by periprocedural myocardial infarction whereas the late outcomes continue to diverge to the end of follow-up. A longer follow-up of these patients may show the emergence of a significant difference in favour of the invasive approach (Doshi P). The ISCHEMIA trial supports either an invasive or conservative approach patients with stable disease who have either no symptoms or mild symptoms that can be controlled with medication and moderate-to-severe ischaemia (Stone G).

\section{ORBITA}

The consistency of the findings in these trials raises the question of what benefit percutaneous intervention offers in patients who have chronic coronary syndrome without disabling symptoms and in whom left main stem stenosis has been excluded. Of course, it is possible that patients with a poor prognosis may be overlooked when a conservative strategy with optimal medical therapy is adopted. The significance of a coronary stenosis seen an angiogram has been open to question for many years. The measurement of the Fractional Flow Rate offers a means to assess the functional significance of an observed stenosis. ${ }^{(15)}$ Several studies have demonstrated that the importance of the stenosis is frequently overestimated or underestimated when assessed on an angiogram. Furthermore, the safety of not treating what appears to be a significant stenosis on angiography when the FFR is within the normal range has been demonstrated repeatedly. Thus, the European Society of Cardiology 2019 Guideline on Chronic Coronary Syndromes recommends that the FFR should be measured in all vessels less than $90 \%$ stenosed and treating only those with an FFR below 0.80 (1) $^{(1)}$

In the ISCHEMIA trial FFR could be used when available and appropriate. However, in the smaller ORBITA trial ${ }^{(16)}$ of only 200 patients, FFR was employed universally. These patients had Canadian Cardiovascular Society class II or III angina. After a period during which medical therapy was optimised, patients underwent assessment of their functional capacity with cardio-pulmonary exercise testing, myocardial ischaemic burden with dobutamine stress echocardiography, and a quality of life assessment. Every patient underwent coronary angiography. All had significant single vessel coronary artery stenosis (mainly left anterior descending) as judged visually from the angiogram. Double-blinded invasive physiological assessment of fractional flow reserve (FFR) and instantaneous wave-free ratio (iFR) was done. The mean FFR was 0.69 , consistent with haemodynamically significant stenoses. Uniquely these patients were randomised to revascularisation with implantation of a drug-eluting stent or to a sham procedure. Blinding was maintained throughout the trial by sedating the patients during the intervention. All patients received guideline-directed 
secondary preventive treatment and similar amounts of antianginal therapy. The guideline-directed therapeutic targets for blood pressure and LDL cholesterol were met. Patients were followed for 6 weeks and then resubmitted to the tests of their functional capacity, ischaemic burden and quality of life. Although there was a significant improvement in the peak stress wall motion score in the stented group, no difference was found in the Duke treadmill score, the trial's primary endpoint, between the 2 groups nor in angina or the degree of ST segment depression after exercise.

The ORBITA trial challenges the concept of a simple linkage between angina pectoris and significant coronary stenosis which is so frequently held by clinicians and their patients and which is moreover biologically plausible. This view of the pathophysiology ignores the possible contributions of symptoms arising from a non-target stenosis and/or from microvascular disease. While the short duration of the ORBITA trial cannot answer questions about potential differences in longer term outcomes of stenting added to medical therapy versus medical therapy alone, it does provide clear insight into a potent, previously unexplored placebo effect in patients who undergo stenting for stable angina.

\section{CONCLUSIONS}

The trials and analyses referred to here include only those patients with chronic coronary syndrome and exclude those with current or recent acute coronary syndromes, left main

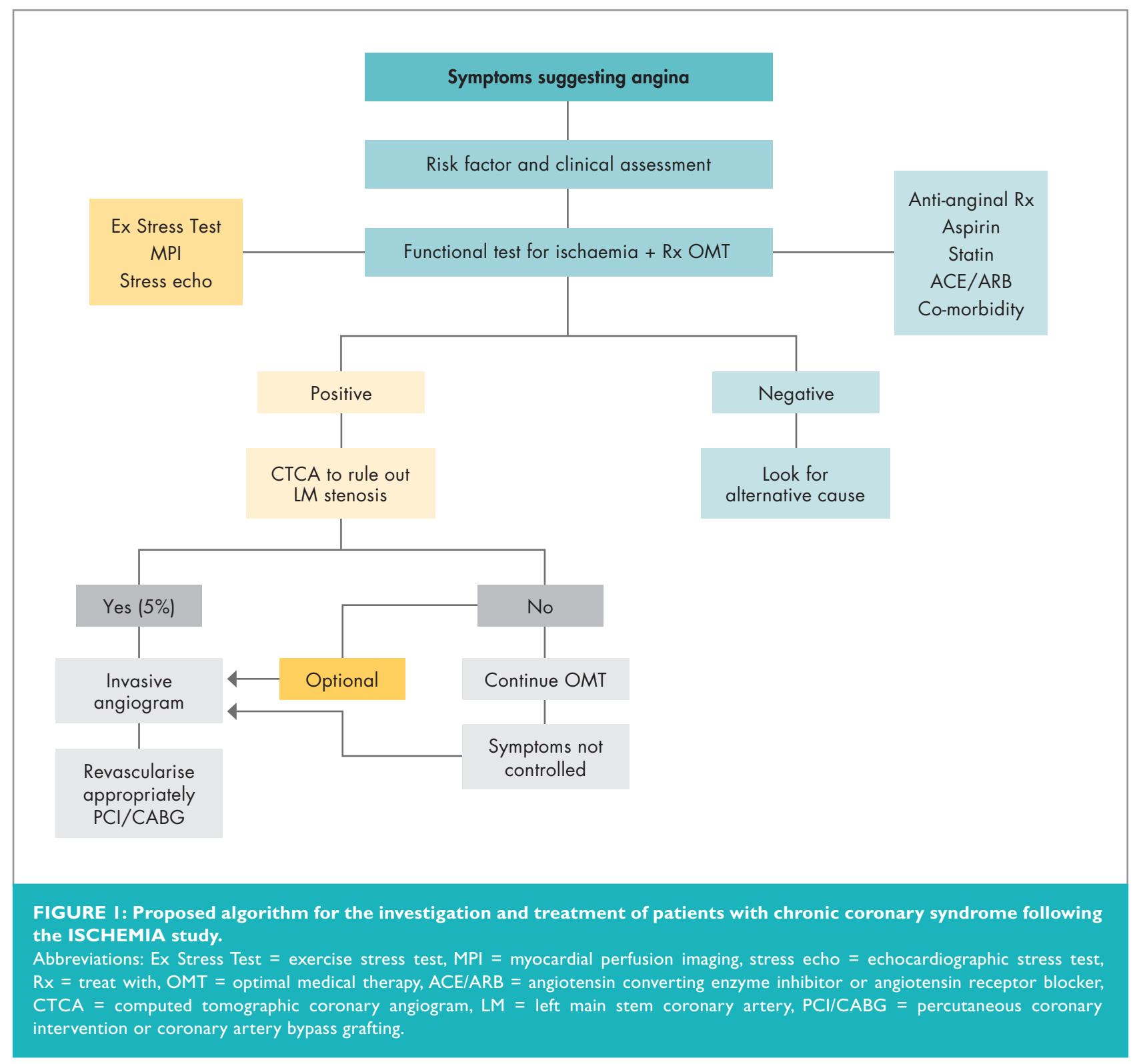


stem stenosis, underlying left-ventricular dysfunction and, in some cases, multivessel coronary artery disease.

For the clinician having to deal with the individual patient and appreciating the modest benefits of percutaneous intervention, it will be important in future to consider whether these trial results are strictly applicable to the specific patient, what the severity or frequency of their angina is, whether there is evidence of significant disease to warrant intervention, as well as taking into account the patient's preference whether for initial medical therapy or revascularisation.

Building on the findings in these trials, an algorithm for managing chronic coronary syndrome is proposed (Figure I). If symptoms suggest ischaemia, whether angina or dyspnoea, the patient should undergo risk factor and clinical assessment. Thereafter a functional test for ischaemia should be performed and intensive guideline-directed optimal medical therapy commenced. If no ischaemia is detected, the patient should be investigated for an alternative cause of the symptoms. If ischaemia is demonstrated, the patient should undergo CT coronary angiography to rule out left main stem stenosis which can be anticipated in about $5 \%$ of patients. Those found to have left main stem stenosis should be submitted to an invasive coronary angiogram and revascularised appropriately with either percutaneous intervention or coronary bypass surgery. Should there be no evidence of left main stem stenosis, it is safe to continue optimal medical therapy only and withhold invasive angiography until medical therapy is judged to have failed. Should the patient favour immediate intervention rather than optimal medical treatment, she/he should be fully informed of the equipoise in survival outcomes between the 2 options.

\section{Conflict of interest: none declared.}

\section{REFERENCES}

I. Knuuti J. Wijns W, Saraste A, et al. 2019 ESC Guidelines for the diagnosis and management of chronic coronary syndromes. Eur Heart J. 2020;4 I:407477. DOI: 10.1093/eurheartj/ehz425.

2. Gruntzig A. Transluminal dilation of coronary artery stenosis. Lancet. 1978: 31 I:263. DOI: 10.1016/50 I40-6736(78)90500-7.

3. CASS Principal Investigators and their Associates. Coronary Artery Surgery Study (CASS): A randomised trial of coronary artery bypass surgery. Survival data. Circulation. 1983:68:939-950. DOI:10.1 | 6 I/01.cir.68.5.939.

4. Chaitman BR, Fisher MD, Bourassa MG, et al. Effect of coronary bypass surgery on survival patterns in subsets of patients with left main coronary artery disease. Report of the Collaborative Study in Coronary Artery Surgery (CASS). Am J Cardiol. 198I;48:765-777. DOI: 10.1016/00029149(8I)90156-9

5. Takaro T, Peduzzi P, Detre KM, et al. Survival in subgroups of patients with left main coronary artery disease. Veterans Administration cooperative study of surgery for coronary arterial occlusive disease circulation. 1982;66: I 4-22. DOI: $10.1161 / 01$.cir.66.1.14.

6. European Coronary Surgery Study Group. Prospective randomised study of coronary artery bypass surgery in stable angina pectoris. Second interim report by the European Coronary Surgery Study Group. Lancet. 1980;2: 49।-495. DOI: 10.1016/S0140-6736(80)91829-2.

7. Boden WE, O'Rourke RA, Teo KK, et al. for the COURAGE Trial Research Group. Optimal medical therapy with or without PCl for stable coronary disease. N Engl J Med. 2007;356: I 503- I I I6. DOI: I0. I056/NEJMoa070829.

8. Frye RL, August P, Brooks MM, et al. for The BARI 2D Study Group. A randomised trial of therapies for type 2 diabetes and coronary artery disease. N Engl J Med. 2009;360:2503- I5. DOI: I0.1056/NEJMoa0805796.

9. Ikeno F, Brooks MM, Nakagawa K, et al. SYNTAX score and long-term outcomes: The BARI-2D trial. J Am Coll Cardiol. 2017;69:395-403. DOI: 10.1016/j.jacc.2016.10.067.

10. Sianos G, Morel MA, Kappetein AP, et al. The SYNTAX score: An angiographic tool grading the complexity of coronary artery disease. Eurolnterv. 2005;2:219-227. PMID:19758907.

II. Windecker S, Stortecky S, Stefanini GG, et al. Revascularisation versus medical treatment in patients with stable coronary artery disease: Network Meta-Analysis. Brit Med J. 2014;348:g3859. DOI: 10.1 I36/bmj.g3859.

12. Maron DJ, Hochman JS, Reynolds HR, et al. Initial invasive or conservative strategy for stable coronary disease. N Engl J Med. 2020;382:| 395-I 407. DOI: 10.1056/NEJMoal915922

13. Bangalore S, Maron DJ, O'Brien SM, et al. Management of coronary disease in patients with advanced kidney disease. N Engl J Med. 2020;382:1608|6|8. DOI: 10.1056/NEJMoal915925.

14. Spertus JA. Jones PG Maron DJ, et al. Health-status outcomes with invasive or conservative care in coronary disease N Engl J Med. 2020;382:1408-1419. DOI: 10.1056/NEJMoal916370.

15. Tonino PA, De Bruyne B, Pijls NH, et al. Fractional flow reserve versus angiography for guiding percutaneous coronary intervention. N Engl J Med. 2009;360:2 |3-24. DOI: I0. I056/NEJMoa08076। I.

16. Al-Lamee R, Thompson D, Dehbi HM, et al. Percutaneous coronary intervention in stable angina (ORBITA): A double-blind, randomised controlled trial. Lancet. 20 I8;39|:3 I-40. DOI: I0.10 |6/ S0 I40-6736(I7)327 |4-9. 